ANNALES

POLONICI MATHEMATICI

$91.1(2007)$

\title{
Integral transforms of functions with restricted derivatives
}

\author{
by Johnny E. Brown (West Lafayette, IN)
}

\begin{abstract}
We show that functions whose derivatives lie in a half-plane are preserved under the Pommerenke, Chandra-Singh, Libera, Alexander and Bernardi integral transforms. We determine precisely how these transforms act on such functions. We prove that if the derivative of a function lies in a convex region then the derivative of its Pommerenke, Chandra-Singh, Libera, Alexander and Bernardi transforms lie in a strictly smaller convex region which can be determined. We also consider iterates of these transforms. Best possible results are obtained.
\end{abstract}

1. Introduction. Let $A(\mathbb{D})$ denote the class of functions $f$ which are analytic in the unit disk $\mathbb{D}$ and normalized by $f(0)=0$ and $f^{\prime}(0)=1$. The classical family of univalent functions in $A(\mathbb{D})$ is denoted by $S$. The following are well-known integral transforms on $A(\mathbb{D})$ :
$\mathbf{A} f(z)=\int_{0}^{z} \frac{f(\zeta)}{\zeta} d \zeta$
(Alexander transform [1]),
$\mathbf{L} f(z)=\frac{2}{z} \int_{0}^{z} f(\zeta) d \zeta$
(Libera transform [9]),
$\mathbf{B}_{c} f(z)=(c+1) \int_{0}^{1} t^{c-1} f(t z) d t, \quad c>-1$
(Bernardi transform [2]).

The Alexander and Libera transforms are special cases of the Bernardi transform with $c=0$ and $c=1$, respectively.

Biernacki [3] claimed that the Alexander transform preserved the class $S$, however a counterexample to this was constructed by Krzyż and Lewandowski [8]. Campbell and Singh [4] later showed that $S$ is not preserved under the Libera transform either. Hence it was of interest to determine which subclasses of $S$ and, more generally, of $A(\mathbb{D})$ are preserved under these and other transforms. It is known that the subclasses of $S$ consisting of convex, starlike and close-to-convex functions (denoted by $K, S^{*}$ and $C$,

2000 Mathematics Subject Classification: Primary 30C45; Secondary 30C75.

Key words and phrases: bounded turning, integral transforms, extreme points. 
respectively) are each preserved under the Alexander and Libera transforms and also under the Bernardi transform for $c=0,1, \ldots$ (see [2] for example). Ruscheweyh and Sheil-Small [13] also proved these same results using the theory of convolutions.

Another interesting integral transform was first introduced by Pommerenke [11]:

$$
\mathbf{P} f(z)=\int_{0}^{z} \frac{f\left(z_{1} \zeta\right)-f\left(z_{2} \zeta\right)}{z_{1} \zeta-z_{2} \zeta} d \zeta
$$

for fixed $\left|z_{1}\right| \leq 1$ and $\left|z_{2}\right| \leq 1$. He proved that if $f \in C(\alpha)$ for $0 \leq \alpha \leq 1$, the class of strongly close-to-convex functions of order $\alpha$ (i.e., $\left|\arg \left\{f^{\prime}(z) / h^{\prime}(z)\right\}\right|$ $\leq \pi \alpha / 2$ for some convex function $h)$, then $\mathbf{P} f \in C(\alpha)$. Note that $\alpha=0$ and $\alpha=1$ correspond to the class of convex and close-to-convex functions, respectively. Recall that a function $f$ is close-to-convex of order $\alpha$ if $\operatorname{Re}\left\{f^{\prime}(z) / h^{\prime}(z)\right\}>\alpha$.

Later, and apparently unaware of this result, Chandra and Singh [5] introduced a special case of the transform (1.1) defined by

$$
\mathbf{P}_{\nu_{1}, \nu_{2}} f(z)=\frac{1}{e^{i \nu_{1}}-e^{i \nu_{2}}} \int_{0}^{z} \frac{f\left(t e^{i \nu_{1}}\right)-f\left(t e^{i \nu_{2}}\right)}{t} d t,
$$

where $0 \leq \nu_{1}<\nu_{2}<2 \pi$, and proved that convex, starlike and close-toconvex functions of order $\alpha$ as well as strongly close-to-convex functions of order $\alpha$ are all preserved under the transform $\mathbf{P}_{\nu_{1}, \nu_{2}}$. Since integral transforms tend to smooth functions these results are not too surprising. In this paper we shall study these transforms on classes of functions in $A(\mathbb{D})$ with restricted derivatives.

A function $f \in A(\mathbb{D})$ is said to be of bounded turning of order $\beta$, where $0 \leq \beta<1$, if $\operatorname{Re}\left\{f^{\prime}(z)\right\}>\beta$ for all $z \in \mathbb{D}$. We denote this class by $R_{\beta}$. By the Noshiro-Warschawski theorem we know that $R_{\beta}$ is a subclass of $S$ and is in fact a subclass of close-to-convex functions (see Duren [6]). It is easy to see that the Bernardi transform maps $R_{\beta}$ into $R_{\beta}$ :

$$
\operatorname{Re}\left\{\left(\mathbf{B}_{c} f\right)^{\prime}(z)\right\}=(c+1) \int_{0}^{1} t^{c} \operatorname{Re}\left\{f^{\prime}(t z)\right\} d t>(c+1) \int_{0}^{1} t^{c} \beta d t=\beta .
$$

It is also known for example that if $f \in R_{0}$ then $\mathbf{A} f \in S^{*}$ (see [14]).

Ponnusamy and Rønning [12] generalized $R_{\beta}$ and studied the Bernardi transform of functions in $A(\mathbb{D})$ whose derivatives lie in an arbitrary halfplane. They defined this class of functions as

$$
\mathcal{P}_{\beta}=\left\{f \in A(\mathbb{D}): \exists \alpha \in \mathbb{R}, \operatorname{Re}\left[e^{i \alpha}\left(f^{\prime}(z)-\beta\right)\right]>0, \forall z \in \mathbb{D}\right\},
$$

where $\beta \in \mathbb{R}$, and proved a number of sharp results including finding the largest $\beta=\beta(c, \gamma)$ such that if $f \in \mathcal{P}_{\beta}$, then its Bernardi transform $\mathbf{B}_{c} f(z)$ 
is starlike of order $\gamma$, generalizing the result in [14]. We should point out that unlike $R_{\beta}$, the class $\mathcal{P}_{\beta}$ may contain non-univalent functions, as can be shown by the function $f(z)=z+z^{2}$ which belongs to every $\mathcal{P}_{\beta}$ for $\beta<-1$, but does not belong to $S$.

We define the class of functions $R_{\beta}^{\alpha}$ as follows:

$$
R_{\beta}^{\alpha}=\left\{f \in A(\mathbb{D}): \operatorname{Re}\left[e^{i \alpha}\left(f^{\prime}(z)-\beta\right)\right]>0, \forall z \in \mathbb{D}\right\} .
$$

It is clear that if $f \in R_{\beta}^{\alpha}$ then $f^{\prime}(0)=1$ and so necessarily we must have

$$
(1-\beta) \cos \alpha>0 \text {. }
$$

Note that for a fixed $\beta$, we have $R_{\beta}^{\alpha} \subset \mathcal{P}_{\beta}$. As above, it is easy to see that the Bernardi transform also maps $R_{\beta}^{\alpha}$ into $R_{\beta}^{\alpha}$. It is natural to ask if the class $R_{\beta}^{\alpha}$ is preserved under the Chandra-Singh transform (1.2) and more generally the Pommerenke transform (1.1). We prove that this is indeed the case and also show that all these transforms actually map $R_{\beta}^{\alpha}$ into strictly smaller subclasses which can be determined.

We can now state our main results.

Theorem 1. Let $\alpha, \beta \in \mathbb{R}$ satisfy (1.4). If $f \in R_{\beta}^{\alpha}$, then:

(a) $\mathbf{P} f \in R_{\beta_{\mathbf{P}}}^{\alpha}$, where

$$
\begin{aligned}
\mathbf{P} f(z) & =\int_{0}^{z} \frac{f\left(z_{1} \zeta\right)-f\left(z_{2} \zeta\right)}{z_{1} \zeta-z_{2} \zeta} d \zeta \quad\left(z_{1}, z_{2} \in \overline{\mathbb{D}}\right), \\
\beta_{\mathbf{P}} & =2 \beta-1+(1-\beta) \frac{3+\delta}{2+2 \delta}
\end{aligned}
$$

and $\delta=\max \left\{\min \left\{\left|z_{1}\right|,\left|z_{2}\right|\right\},\left|z_{1}+z_{2}\right| / 2\right\}$.

(b) $\mathbf{P}_{\nu_{1}, \nu_{2}} f \in R_{\beta_{*}}^{\alpha}$, where

$$
\mathbf{P}_{\nu_{1}, \nu_{2}} f(z)=\frac{1}{e^{i \nu_{1}}-e^{i \nu_{2}}} \int_{0}^{z} \frac{f\left(t e^{i \nu_{1}}\right)-f\left(t e^{i \nu_{2}}\right)}{t} d t \quad\left(0 \leq \nu_{1}<\nu_{2}<2 \pi\right),
$$

$$
\beta_{*}=2 \beta-1+(1-\beta) \nu / \sin \nu
$$

and $\nu=\frac{1}{2} \min \left\{\left(\nu_{2}-\nu_{1}\right), 2 \pi-\left(\nu_{2}-\nu_{1}\right)\right\} \in(0, \pi / 2]$. This result is best possible.

(c) $\mathbf{B}_{c} f \in R_{\beta_{c}}^{\alpha}$ for $c=0,1, \ldots$, where

$$
\begin{aligned}
\mathbf{B}_{c} f(z) & =(c+1) \int_{0}^{1} t^{c-1} f(t z) d t, \\
\beta_{c} & =2 \beta-1+(1-\beta) \gamma_{c}
\end{aligned}
$$


with $\gamma_{0}=\log 4$ and

$$
\gamma_{c}=2(c+1)(-1)^{c}\left[\log 2-\sum_{k=1}^{c} \frac{(-1)^{k+1}}{k}\right], \quad c=1,2, \ldots,
$$

and $1<\gamma_{c}<2$. This result is best possible.

Remark 1. If both $z_{1}$ and $z_{2}$ lie on $|z|=1$, then the Pommerenke transform (1.1) reduces to the Chandra-Singh transform (1.2). Consequently, without loss of generality, we shall henceforth assume when referring to the Pommerenke transform that at most one of $z_{1}$ and $z_{2}$ lies on $|z|=1$. Thus we then have $0 \leq \delta<1$.

The proof of Theorem 1 is given in the next section. We first state and prove some applications.

Corollary 1. If $f \in R_{\beta}^{\alpha}$, then:

(i) $\mathbf{P} f \in R_{\beta_{\mathbf{P}}}^{\alpha} \subset R_{\beta}^{\alpha}$, where $\beta_{\mathbf{P}}$ is given by (1.5).

(ii) $\mathbf{P}_{\nu_{1}, \nu_{2}} f \in R_{\beta_{*}}^{\alpha} \subset R_{\beta}^{\alpha}$, where $\beta_{*}$ is given by (1.6).

(iii) $\mathbf{B}_{c} f \in R_{\beta_{c}}^{\alpha} \subset R_{\beta}^{\alpha}$ for $c=0,1, \ldots$, where $\beta_{c}$ is given by (1.7).

Proof. Let $\alpha$ and $\beta$ be fixed and let

$$
\beta^{* *}=2 \beta-1+M(1-\beta),
$$

where $M>1$ is fixed. We assert that $R_{\beta^{* *}}^{\alpha} \subset R_{\beta}^{\alpha}$. The corollary then follows because if $f \in R_{\beta}^{\alpha}$ then from the theorem in each of the cases (i)-(iii) we simply let $M=(3+\delta) /(2+2 \delta), \nu / \sin \nu, \gamma_{c}$, respectively, to conclude that the corresponding transform $F$ belongs to $R_{\beta^{* *}}^{\alpha}$.

To prove our assertion that $R_{\beta^{* *}}^{\alpha} \subset R_{\beta}^{\alpha}$ we consider several cases. Suppose $F \in R_{\beta^{* *}}^{\alpha}$ and recall that $(1-\beta) \cos \alpha>0$.

CASE 1: $-\infty<\beta<1$. In this case we have $\cos \alpha>0$ and we obtain

$$
\beta^{* *}=2 \beta-1+M(1-\beta)>\beta \text {. }
$$

Since $F \in R_{\beta^{* *}}^{\alpha}$, i.e., $\operatorname{Re}\left\{e^{i \alpha}\left[F^{\prime}(z)-\beta^{* *}\right]\right\}>0$, we obtain

$$
\operatorname{Re}\left\{e^{i \alpha} F^{\prime}(z)\right\}>\beta^{* *} \cos \alpha>\beta \cos \alpha,
$$

which implies that $F \in R_{\beta}^{\alpha}$.

CASE 2: $1<\beta<\infty$. Here $\cos \alpha<0$ and observe that $\beta^{* *}<\beta$. Thus we have $\operatorname{Re}\left\{e^{i \alpha} F^{\prime}(z)\right\}>\beta^{* *} \cos \alpha>\beta \cos \alpha$ and hence $F \in R_{\beta}^{\alpha}$.

In the above result, these transforms map $R_{\beta}^{\alpha}$ into strictly smaller subclasses, and since the values given by (1.6) and (1.7) are best possible, the Chandra-Singh and Bernardi transforms do not map $R_{\beta}^{\alpha}$ into any class smaller than the corresponding $R_{\beta^{* *}}^{\alpha}$. 
If the derivative of an arbitrary function in $A(\mathbb{D})$ lies in a region, then one might expect that the region in which the derivative of its integral transform lies should be related. We obtain the following result:

Theorem 2. Let $f \in A(\mathbb{D})$ and let $F$ be its Pommerenke, ChandraSingh or Bernardi transform with $c=0,1, \ldots$ If $\Delta(f)=\left\{f^{\prime}(z): z \in \mathbb{D}\right\}$ lies in a convex region $\Omega$, then $\Delta(F)=\left\{F^{\prime}(z): z \in \mathbb{D}\right\}$ also lies in $\Omega$.

Proof. Note that $f \in R_{\beta}^{\alpha}$ if and only if $f(r z) / r \in R_{\beta}^{\alpha}$ for any $0<$ $r<1$. Hence, without loss of generality, we may assume that $\Omega \subset \mathbb{C}$ is bounded. Furthermore, we may assume that $\Omega$ is a convex polygonal region. Consequently, it is sufficient to prove the theorem when $\Omega$ is a bounded convex polygonal region with $m$ sides. Necessarily we have $1 \in \Omega$. Let $f \in$ $A(\mathbb{D})$ and suppose that $\Delta(f)=\left\{f^{\prime}(z): z \in \mathbb{D}\right\} \subset \Omega$.

Assume first that $\partial \Omega$ contains no horizontal segments. Because $\bar{\Omega}$ may be obtained as the intersection of $m$ closed half-planes, each containing 1 , it follows that

$$
f \in \bigcap_{j=1}^{m} R_{\beta_{j}}^{\alpha_{j}}
$$

for suitable choices of $\alpha_{j}$ and $\beta_{j}$, each satisfying $\left(1-\beta_{j}\right) \cos \alpha_{j}>0$. To see this, we let $L_{j}$ be the line bounding a side of $\Omega, \beta_{j}$ its intersection with the real axis and $\mu_{j}\left(0<\mu_{j}<\pi\right)$ the angle $L_{j}$ makes with the positive real axis. If $\beta_{j}>1$, choose $\alpha_{j}=3 \pi / 2-\mu_{j}$, while if $\beta_{j}<1$, set $\alpha_{j}=\pi / 2-\mu_{j}$. Hence $f \in R_{\beta_{j}}^{\alpha_{j}}$ for each $j$ and by Corollary 1 the same holds for $F$. Thus $F \in \bigcap_{j=1}^{m} R_{\beta_{j}}^{\alpha_{j}}$ and so we conclude that $\Delta(F) \subset \Omega$.

If a side of $\bar{\Omega}$ is a horizontal segment then we construct a larger convex polygonal region containing all non-horizontal sides of $\Omega$ but replace each horizontal side by two non-horizontal sides as follows. Let $0<\varepsilon<1$ and define the convex set $\Omega(\varepsilon)$ to be bounded by all the lines bounding $\bar{\Omega}$ except the horizontal lines. Each horizontal line is to be replaced by two intersecting lines. In particular, if say $\Omega$ is bounded by the horizontal line $L_{h}$ through the vertices $\omega_{1}=a+i \lambda$ and $\omega_{2}=b+i \lambda$ with $a<b$ and $\lambda>0$, then instead of bounding $\Omega(\varepsilon)$ by $L_{h}$, we bound it by the two lines $L_{h}^{(1)}$ and $L_{h}^{(2)}$ which pass through the pair $\omega_{1}$ and $\omega_{\varepsilon}=(b+a) / 2+i[\lambda+\varepsilon(b-a)]$ and the pair $\omega_{2}$ and $\omega_{\varepsilon}$, respectively. With this construction, it is clear that $\Omega \subset \Omega(\varepsilon)$ for all $0<\varepsilon<1$ and that $\Omega(\varepsilon)$ has no horizontal lines bounding it. A similar construction holds for $\lambda<0$. Apply the above argument to $\Omega(\varepsilon)$ and let $\varepsilon \rightarrow 0$ to complete the proof of the theorem.

REMARK 2. It should be pointed out that by Corollary 1 , since the transforms map $R_{\beta}^{\alpha}$ strictly into itself, we actually have $\Delta(F) \subset \Omega^{\prime} \subset \Omega$, 
where $\Omega^{\prime}$ is a convex region strictly inside $\Omega$. The convex region $\Omega^{\prime}$ can be determined, once $\Omega$ is known.

Finally, we consider iterates of integral transforms. Because these integral transforms map $R_{\beta}^{\alpha}$ into strictly smaller subclasses the following result obtains:

Theorem 3. If $f$ is an arbitrary function in $R_{\beta}^{\alpha}$ and $\mathbf{T} f$ is its Pommerenke, Chandra-Singh or Bernardi transform with $c=0,1, \ldots$, then

$$
\lim _{n \rightarrow \infty} \mathbf{T}^{(n)} f(z)=z,
$$

where $\mathbf{T}^{(n)}=\mathbf{T} \circ \cdots \circ \mathbf{T}$ is the $n^{\text {th }}$ iterate of $\mathbf{T}$ and the convergence is uniform on compact subsets in $\mathbb{D}$.

We shall also prove this theorem in the next section.

2. Proof of the main results. We begin with a few preliminaries about the class $R_{\beta}^{\alpha}$. Assume throughout that $\alpha$ and $\beta$ are fixed and satisfy (1.4).

It is clear that the function $K$ defined by

$$
K(z)=e^{-i \alpha}[A z+B \log (1-z)],
$$

where

$$
A=-\lambda \cos \alpha+i \sin \alpha, \quad B=-(1+\lambda) \cos \alpha, \quad \lambda=1-2 \beta
$$

belongs to the class $R_{\beta}^{\alpha}$ and so it is non-empty. The class $R_{\beta}^{\alpha}$ is convex: if $f, g \in R_{\beta}^{\alpha}$ then $t f+(1-t) g \in R_{\beta}^{\alpha}$ for all $0 \leq t \leq 1$. It is also rotationally invariant: $f \in R_{\beta}^{\alpha}$ if and only if $e^{-i \mu} f\left(e^{i \mu} z\right) \in R_{\beta}^{\alpha}$ for $\mu \in \mathbb{R}$.

The Carathéodory class $\mathfrak{P}$ consists of all functions $p$ which are analytic in $\mathbb{D}$ with $\operatorname{Re} p(z)>0$ and normalized by $p(0)=1$. Observe that $g \in R_{\beta}^{\alpha}$ if and only if

$$
p(z)=\frac{e^{i \alpha}\left(g^{\prime}(z)-\beta\right)-i(1-\beta) \sin \alpha}{(1-\beta) \cos \alpha}
$$

belongs to $\mathfrak{P}$. From this and the distortion theorems for $p \in \mathfrak{P}$ (see [6] or [7] for example), we see that if $g \in R_{\beta}^{\alpha}$, then $\left|g^{\prime}(z)\right|$ and hence $|g(z)|$ are bounded on all compact sets in $\mathbb{D}$, and so the normalization for functions in $R_{\beta}^{\alpha}$ makes it a compact family.

The extreme points of the Carathéodory class $\mathfrak{P}$ are well-known [7]:

$$
\mathcal{E}(\mathfrak{P})=\left\{\frac{1+x z}{1-x z}:|x|=1\right\} .
$$

From (2.3) and (2.4) it follows that the extreme points for the class $R_{\beta}^{\alpha}$ are precisely

$$
\mathcal{E}\left(R_{\beta}^{\alpha}\right)=\{\bar{x} K(x z):|x|=1\}
$$

where $K$ is defined by (2.1) and (2.2). 
We will make use of the following result, essentially due to Marx [10].

LEMma 1. If

$$
H(\theta, \mu)=\operatorname{Im}\left\{-e^{-i \theta} \log \frac{1-e^{i(\theta+\mu)}}{1-e^{i(\theta-\mu)}}\right\}, \quad 0 \leq \theta, \mu \leq \pi,
$$

then

$$
\min _{0 \leq \theta \leq \pi} H(\theta, \mu)= \begin{cases}\mu, & 0 \leq \mu \leq \pi / 2, \\ \pi-\mu, & \pi / 2<\mu \leq \pi\end{cases}
$$

Proof. Observe that if $\theta \neq \mu$ then

$$
H(\theta, \mu)=\frac{\sin \theta}{2} \log \frac{1-\cos (\theta+\mu)}{1-\cos (\theta-\mu)}-\gamma \cos \theta,
$$

where

$$
\gamma= \begin{cases}\mu, & 0 \leq \mu<\theta \leq \pi \\ \mu-\pi, & 0 \leq \theta<\mu \leq \pi\end{cases}
$$

After a calculation we obtain

$$
\frac{\partial H}{\partial \theta}=\frac{\cos \theta}{2} \log \frac{1-\cos (\theta+\mu)}{1-\cos (\theta-\mu)}+\frac{\sin \theta \sin \mu}{\cos \theta-\cos \mu}+\gamma \sin \theta .
$$

A further calculation leads to

$$
\begin{aligned}
\frac{\partial}{\partial \mu}\left(\frac{\partial H}{\partial \theta}\right) & =\frac{\sin \theta}{(\cos \theta-\cos \mu)^{2}}\left(2 \cos \theta \cos \mu-\cos ^{2} \theta-1\right) \\
& \leq-\frac{(\sin \theta)(1-|\cos \theta|)^{2}}{(\cos \theta-\cos \mu)^{2}}
\end{aligned}
$$

Consequently, for fixed $0 \leq \theta_{0} \leq \pi$, the function $\partial H / \partial \theta$ is non-increasing with $\mu$.

Suppose first that $0 \leq \theta_{0}<\mu \leq \pi$. Then

$$
\frac{\partial H}{\partial \theta}\left(\theta_{0}, \mu\right) \geq \frac{\partial H}{\partial \theta}\left(\theta_{0}, \pi\right)=0
$$

and so for $0 \leq \theta<\mu \leq \pi$, we see that $H$ is a non-decreasing function of $\theta$ and thus

$$
H(\theta, \mu) \geq H(0, \mu)=\pi-\mu .
$$

Next, if $0 \leq \mu<\theta_{0} \leq \pi$ then

$$
\frac{\partial H}{\partial \theta}\left(\theta_{0}, \mu\right) \leq \frac{\partial H}{\partial \theta}\left(\theta_{0}, 0\right)=0
$$

In this case, $H$ is a non-increasing function of $\theta$ and hence for $0 \leq \mu<\theta \leq \pi$ we get

$$
H(\theta, \mu) \geq H(\pi, \mu)=\mu .
$$

Thus if $\theta \neq \mu$ then $H(\theta, \mu) \geq \min \{\mu,(\pi-\mu)\}$ and the function is unbounded as $\theta \rightarrow \mu$. This proves the lemma. 
It should be pointed out that there is a typo in formula (65) in Marx [10]. It should read:

$$
\frac{\partial}{\partial \phi}\left(4 \sin \phi \frac{\partial p(\phi, \theta)}{\partial \theta}\right)=\frac{(2 \sin \theta)\left(2 \cos \theta \cos \phi-\cos ^{2} \theta-1\right)}{(\cos \theta-\cos \phi)^{2}} .
$$

Fortunately, his conclusion that the function on the left is non-positive still holds as our (2.6) shows.

LEMma 2. If

$$
\Phi\left(\zeta_{1}, \zeta_{2}\right)=\frac{1}{\zeta_{2}-\zeta_{1}} \log \frac{1-\zeta_{1}}{1-\zeta_{2}}
$$

and $\zeta_{1}, \zeta_{2} \in \overline{\mathbb{D}}\left(\zeta_{1} \neq \zeta_{2}\right)$, then

$$
\operatorname{Re} \Phi\left(\zeta_{1}, \zeta_{2}\right) \geq \frac{3+\delta}{4+4 \delta}
$$

where $\delta=\max \left\{\min \left\{\left|\zeta_{1}\right|,\left|\zeta_{2}\right|\right\},\left|\zeta_{1}+\zeta_{2}\right| / 2\right\}$.

Proof. Let $\omega(t)=\zeta_{1}+\left(\zeta_{2}-\zeta_{1}\right) t, 0 \leq t \leq 1$, be the line segment from $\zeta_{1}$ to $\zeta_{2}$ in $\overline{\mathbb{D}}$. It follows that $|\omega(t)| \leq \delta$ for $0 \leq t \leq 1 / 2$ or $1 / 2 \leq t \leq 1$. To see this, suppose say $\delta=\left|\zeta_{1}\right|$; then

$$
|\omega(1 / 2)|=\left|\zeta_{1}+\zeta_{2}\right| / 2 \leq\left|\zeta_{1}\right|=|\omega(0)|=\delta
$$

and hence $|\omega(t)| \leq \delta$ for $0 \leq t \leq 1 / 2$. The proof of the other cases follows a similar pattern. Using this we conclude that

$$
\begin{aligned}
\operatorname{Re} \Phi\left(\zeta_{1}, \zeta_{2}\right) & =\operatorname{Re}\left\{\frac{1}{\zeta_{2}-\zeta_{1}} \int_{\zeta_{1}}^{\zeta_{2}} \frac{1}{1-z} d z\right\}=\operatorname{Re} \int_{0}^{1} \frac{1}{1-\omega(t)} d t \\
& \geq \int_{0}^{1} \frac{1}{1+|\omega(t)|} d t \geq \frac{1}{2} \cdot \frac{1}{2}+\frac{1}{2} \cdot \frac{1}{1+\delta}=\frac{3+\delta}{4+4 \delta} .
\end{aligned}
$$

We can now prove the main results.

Proof of Theorem 1. We consider each transform separately.

(a) Let $F=\mathbf{P} f$. Now for fixed $z_{0} \in \mathbb{D}$ we have

$$
\operatorname{Re}\left\{e^{i \alpha} F^{\prime}\left(z_{0}\right)\right\}=\operatorname{Re}\left\{e^{i \alpha} \frac{f\left(z_{1} z_{0}\right)-f\left(z_{2} z_{0}\right)}{z_{1} z_{0}-z_{2} z_{0}}\right\} .
$$

The linear functional

$$
L(f)=e^{i \alpha} \frac{f\left(z_{1} z_{0}\right)-f\left(z_{2} z_{0}\right)}{z_{1} z_{0}-z_{2} z_{0}}
$$

attains its minimum real part over the set of extreme points of $R_{\beta}^{\alpha}$. (This follows e.g. from Thm. 4.5, p. 44, in [7] by observing that $-\min \operatorname{Re}\{L(f)\}$ $=\max \operatorname{Re}\{J(f)\}$, where $J(f)=-L(f)$.) Consequently,

$$
\operatorname{Re}\left\{e^{i \alpha} F^{\prime}\left(z_{0}\right)\right\} \geq \min _{|x|=1} \operatorname{Re}\left\{e^{i \alpha} \frac{K\left(x z_{1} z_{0}\right)-K\left(x z_{2} z_{0}\right)}{x z_{1} z_{0}-x z_{2} z_{0}}\right\}
$$


where $K$ is given by (2.1). Using (2.1) and (2.2) we obtain

$$
\operatorname{Re}\left\{e^{i \alpha} F^{\prime}\left(z_{0}\right)\right\} \geq \min _{|x|=1} \operatorname{Re}\left\{A+B \frac{1}{\left(x z_{1} z_{0}-x z_{2} z_{0}\right)} \log \frac{1-x z_{1} z_{0}}{1-x z_{2} z_{0}}\right\} .
$$

The above function is analytic in the variable $z=x z_{0}$ and hence by the minimum principle and Lemma 2 we may conclude that

$$
\begin{aligned}
\operatorname{Re}\left\{e^{i \alpha} F^{\prime}\left(z_{0}\right)\right\} & >(2 \beta-1) \cos \alpha+2(1-\beta)(\cos \alpha) \min _{|z|=1} \operatorname{Re}\left\{\Phi\left(\left\{z z_{1}\right\},\left\{z z_{2}\right\}\right)\right\} \\
& \geq(2 \beta-1) \cos \alpha+2(1-\beta)(\cos \alpha) \frac{3+\delta}{4+4 \delta}=\beta_{\mathbf{P}} \cos \alpha .
\end{aligned}
$$

Thus for any $z_{0} \in \mathbb{D}$, we get $\operatorname{Re}\left\{e^{i \alpha}\left[F^{\prime}\left(z_{0}\right)-\beta_{\mathbf{P}}\right]\right\}>0$ and so $F=\mathbf{P} f \in R_{\beta_{\mathbf{P}}}^{\alpha}$.

(b) Let $F=\mathbf{P}_{\nu_{1}, \nu_{2}} f$. Note that the function $F \in R_{\beta_{*}}^{\alpha}$ if and only if $G(z)=e^{-i \mu} F\left(e^{i \mu} z\right) \in R_{\beta_{*}}^{\alpha}$ for any $\mu \in \mathbb{R}$. Hence we see that

$$
G(z)=\frac{1}{e^{i\left(\nu_{1}+\mu\right)}-e^{i\left(\nu_{2}+\mu\right)}} \int_{0}^{z} \frac{f\left(s e^{i\left(\nu_{1}+\mu\right)}\right)-f\left(s e^{i\left(\nu_{2}+\mu\right)}\right)}{s} d s .
$$

If $\nu=\left(\nu_{2}-\nu_{1}\right) / 2$ then setting $\mu=-\left(\nu_{1}+\nu_{2}\right) / 2$ in $(2.7)$ gives

$$
G(z)=\frac{1}{e^{i \nu}-e^{-i \nu}} \int_{0}^{z} \frac{f\left(s e^{i \nu}\right)-f\left(s e^{-i \nu}\right)}{s} d s .
$$

On the other hand, if $\nu=\pi-\left(\nu_{2}-\nu_{1}\right) / 2$, set $\mu=\pi-\left(\nu_{1}+\nu_{2}\right) / 2$ to obtain the same form of $G(z)$. Thus it is sufficient to show that if $f \in R_{\beta}^{\alpha}$, then $G \in R_{\beta_{*}}^{\alpha}$ where

$$
G(z)=\frac{1}{e^{i \nu}-e^{-i \nu}} \int_{0}^{z} \frac{f\left(s e^{i \nu}\right)-f\left(s e^{-i \nu}\right)}{s} d s
$$

with $0<\nu \leq \pi / 2$ and

$$
\beta_{*}=2 \beta-1+(1-\beta) \frac{\nu}{\sin \nu} .
$$

For fixed $0<\nu \leq \pi / 2$ we see from (2.8) that

$$
\operatorname{Re}\left\{e^{i \alpha} G^{\prime}(z)\right\}=\operatorname{Re}\left\{\frac{e^{i \alpha}}{2 i \sin \nu} \frac{f\left(z e^{i \nu}\right)-f\left(z e^{-i \nu}\right)}{z}\right\} .
$$

Now fix $z_{0} \in \mathbb{D}$ and consider the linear functional on $A(\mathbb{D})$ defined by

$$
L(f)=\frac{e^{i \alpha}}{2 i \sin \nu} \frac{f\left(z_{0} e^{i \nu}\right)-f\left(z_{0} e^{-i \nu}\right)}{z_{0}} .
$$

The minimum real part of $L$ is achieved at an extreme point of $R_{\beta}^{\alpha}$. Hence

$$
\operatorname{Re}\left\{e^{i \alpha} G^{\prime}\left(z_{0}\right)\right\} \geq \min _{|x|=1} \operatorname{Re}\{L(\bar{x} K(x z))\}
$$


where $K$ is given by (2.1). A calculation shows that

$$
\{L(\bar{x} K(x z))\}=A+\frac{B}{2 i \sin \nu} \frac{1}{x z_{0}} \log \frac{1-e^{i \nu} x z_{0}}{1-e^{-i \nu} x z_{0}} .
$$

This is an analytic function of $\omega=x z_{0}$. By (2.2) it follows from the minimum principle and symmetry that

$$
\begin{aligned}
\operatorname{Re}\left\{e^{i \alpha} G^{\prime}\left(z_{0}\right)\right\} & \geq \min _{|x|=1} \operatorname{Re}\{L(\bar{x} K(x z))\} \\
& >(2 \beta-1) \cos \alpha+\frac{(1-\beta) \cos \alpha}{\sin \nu} \min _{0 \leq \theta \leq \pi} H(\theta, \nu)
\end{aligned}
$$

where

$$
H(\theta, \nu)=\operatorname{Im}\left\{-e^{-i \theta} \log \frac{1-e^{i(\theta+\nu)}}{1-e^{i(\theta-\nu)}}\right\} .
$$

We may now apply Lemma 1 with $\mu=\nu$ and $0<\nu \leq \pi / 2$ to see that

$$
\operatorname{Re}\left\{e^{i \alpha} G^{\prime}\left(z_{0}\right)\right\} \geq(2 \beta-1) \cos \alpha+(1-\beta)(\cos \alpha) \frac{\nu}{\sin \nu}=\beta_{*} \cos \alpha .
$$

Hence $\operatorname{Re}\left\{e^{i \alpha}\left[G^{\prime}\left(z_{0}\right)-\beta_{*}\right]\right\}>0$ for any $z_{0} \in \mathbb{D}$ and so $G \in R_{\beta_{*}}^{\alpha}$.

To show that $\beta_{*}$ is best possible, consider the function $f=K$ defined in (2.1) and let $z=-r$. A calculation gives

$$
\operatorname{Re}\left\{e^{i \alpha} G^{\prime}(-r)\right\}=\left[2 \beta-1+\frac{1-\beta}{\sin \nu} \operatorname{Im}\left\{\frac{1}{r} \log \frac{1+r e^{i \nu}}{1+r e^{-i \nu}}\right\}\right] \cos \alpha
$$

and hence

$$
\lim _{r \rightarrow 1} \operatorname{Re}\left\{e^{i \alpha}\left[G^{\prime}(-r)-\beta_{*}\right]\right\}=0
$$

(c) Let $F=\mathbf{B}_{c} f$. For $z_{0} \in \mathbb{D}$ arbitrary but fixed, the linear functional $L(f)=(c+1) \int_{0}^{1} e^{i \alpha} t^{c} f^{\prime}\left(t z_{0}\right) d t$ assumes its minimum real part over the set of extreme points of $R_{\beta}^{\alpha}$ and hence

$$
\operatorname{Re}\left\{e^{i \alpha} F^{\prime}\left(z_{0}\right)\right\} \geq \min _{|x|=1} \operatorname{Re}\left\{(c+1) \int_{0}^{1} e^{i \alpha} t^{c} K^{\prime}\left(x t z_{0}\right) d t\right\}
$$

where $K$ is given by (2.1) and (2.2). Next, by the minimum principle, we see that

$$
\begin{aligned}
\min _{|x|=1} \int_{0}^{1} \operatorname{Re}\left\{\frac{t^{c}}{1-t x z_{0}}\right\} d t & >\min _{-\pi<\theta \leq \pi} \int_{0}^{1} \operatorname{Re}\left\{\frac{t^{c}}{1-t e^{i \theta}}\right\} d t \\
& \geq \int_{0}^{1} \frac{t^{c}}{1+t} d t=(-1)^{c}\left[\log 2-\sum_{k=1}^{c} \frac{(-1)^{k+1}}{k}\right] .
\end{aligned}
$$


Using (2.9) and this estimate we obtain after a calculation

$$
\begin{aligned}
& \operatorname{Re}\left\{e^{i \alpha} F^{\prime}\left(z_{0}\right)\right\} \\
& \quad>\left\{2 \beta-1+2(1-\beta)(c+1)(-1)^{c}\left[\log 2-\sum_{k=1}^{c} \frac{(-1)^{k+1}}{k}\right]\right\} \cos \alpha \\
& \quad=\left\{2 \beta-1+(1-\beta) \gamma_{c}\right\} \cos \alpha=\beta_{c} \cos \alpha .
\end{aligned}
$$

(If $c=0$, then $\int_{0}^{1} \operatorname{Re}\left\{1 /\left(1-t e^{i \theta}\right)\right\} d t \geq \log 2$ and from (2.9) we get the above result with $\gamma_{0}=\log 4$.) Thus $\operatorname{Re}\left\{e^{i \alpha}\left[\bar{F}^{\prime}\left(z_{0}\right)-\beta_{c}\right]\right\}>0$ and hence we conclude that $F \in R_{\beta_{c}}^{\alpha}$. Because

$$
\frac{1}{2(c+1)}=\int_{0}^{1} \frac{t^{c}}{2} d t<\int_{0}^{1} \frac{t^{c}}{1+t} d t<\int_{0}^{1} t^{c} d t=\frac{1}{c+1}
$$

and

$$
\int_{0}^{1} \frac{t^{c}}{1+t} d t=(-1)^{c}\left[\log 2-\sum_{k=1}^{c} \frac{(-1)^{k+1}}{k}\right]=\frac{\gamma_{c}}{2(c+1)}
$$

we must have

$$
1<\gamma_{c}<2 \text {. }
$$

To show that $\beta_{c}$ is best possible we consider the function $f=K$ given by (2.1) and let $z=-r$ :

$$
\begin{aligned}
\operatorname{Re}\left\{e^{i \alpha} F^{\prime}(-r)\right\} & =(c+1) \int_{0}^{1} \operatorname{Re}\left\{e^{i \alpha} K^{\prime}(-t r)\right\} d t \\
& =\left[2 \beta-1+2(1-\beta)(c+1) \int_{0}^{1} \frac{t^{c}}{1+r t} d t\right] \cos \alpha .
\end{aligned}
$$

Using this and (1.7) we let $r \rightarrow 1$ to find that $\operatorname{Re}\left\{e^{i \alpha}\left[F^{\prime}(-r)-\beta_{c}\right]\right\} \rightarrow 0$, and hence $\beta_{c}$ is best possible. This completes the proof of Theorem 1 .

Proof of Theorem 3. Fix $\alpha$ and $\beta$ satisfying (1.4). By Corollary 1, we conclude that each of the transforms $\mathbf{P}, \mathbf{P}_{\nu_{1}, \nu_{2}}$ and $\mathbf{B}_{c}($ for $c=0,1, \ldots)$ maps $R_{\beta}^{\alpha}$ into $R_{\beta^{* *}}^{\alpha}$, where

$$
\beta^{* *}=2 \beta-1+M(1-\beta)=\beta(2-M)+M-1
$$

and

$$
M=\frac{3+\delta}{2+2 \delta}, \quad M=\frac{\nu}{\sin \nu} \quad \text { or } \quad M=\gamma_{c},
$$

respectively. Recall that $0 \leq \delta<1$ (see Remark 1) and $0<\nu \leq \pi / 2$. Consequently, in each case we have $1<M<2$. Now let

$$
F_{0}=f, \quad F_{1}=\mathbf{T} f, \quad \ldots, \quad F_{n}=(\mathbf{T} \circ \cdots \circ \mathbf{T}) f,
$$


where $\mathbf{T}$ is the Pommerenke transform $\mathbf{P}$, the Chandra-Singh transform $\mathbf{P}_{\nu_{1}, \nu_{2}}$ or the Bernardi transform $\mathbf{B}_{c}$. For convenience, set $x=2-M$ in (2.10). From Corollary 1, we may apply an induction argument to show that $F_{n} \in R_{\beta(n)}^{\alpha} \subset R_{\beta}^{\alpha}$ where

$$
\beta(n)=\beta x^{n}+1-x^{n} .
$$

Let $\varepsilon>0$ be given. It suffices to show that $\left|F_{n}(z)-z\right|<\varepsilon$ for all $|z| \leq r<1$ and all $n>N(\varepsilon)$. Since $F_{n} \in R_{\beta(n)}^{\alpha}$, it follows from (2.3) that

$$
F_{n}^{\prime}(z)=e^{-i \alpha}\{p(z)-1\}(1-\beta(n)) \cos \alpha+1
$$

for some $p \in \mathfrak{P}$. Using (2.11) and the estimate $\left|p\left(r e^{i \theta}\right)\right| \leq(1+r) /(1-r)$ for any $p \in \mathfrak{P}$, we obtain

$$
\begin{aligned}
\left|F_{n}(z)-z\right| & =\left|\int_{0}^{z}\left[e^{-i \alpha}\{p(\zeta)-1\}(1-\beta(n)) \cos \alpha\right] d \zeta\right| \\
& =\left|z e^{-i \alpha}(1-\beta(n)) \cos \alpha \int_{0}^{1}\{p(t z)-1\} d t\right| \\
& \leq r(1-\beta(n)) \cos \alpha \int_{0}^{1}\left\{\frac{2}{1-r t}\right\} d t \\
& =x^{n}\{-2(1-\beta)(\cos \alpha) \log (1-r)\} .
\end{aligned}
$$

Hence, since $0<x<1$, by choosing $n$ sufficiently large we obtain the desired estimate, and this completes the proof of Theorem 3.

3. Remarks. (1) Our results show that the Pommerenke, ChandraSingh and Bernardi transforms map $R_{\beta}^{\alpha}$ into strictly smaller classes. It is not too difficult to see that these transforms map $K, S^{*}$ and $C$ into smaller classes but these subclasses are not given explicitly as we have for $R_{\beta}^{\alpha}$. It is known however that the Alexander transform maps $S^{*}$ one-to-one and onto $K$, i.e., $f \in S^{*}$ if and only if $\mathbf{A} f \in K$. This is in fact Alexander's original theorem in [1].

(2) The search for invariant subclasses under these transforms stemmed from the fact that $S$ was not preserved under $\mathbf{L}$ or $\mathbf{A}$. The Chandra-Singh transform does not preserve $S$ either. In fact, simply consider the spirallike function in $S$ given in [8]:

$$
f(z)=\frac{z}{(1-i z)^{1-i}}
$$

where the principal branch of $(1-i z)^{1-i}$ is chosen. If we let $\nu_{1}=0$ and $\nu_{2}=\pi$ and apply (1.2) to this $f$, then

$$
F(z)=\mathbf{P}_{0, \pi} f(z)=\frac{1}{2}\left\{e^{i \log (1-i z)}-e^{i \log (1+i z)}\right\} .
$$


A check shows that for all $k \in \mathbb{N}$, we get $F\left(z_{k}\right)=0$ where

$$
z_{k}=i \frac{1-e^{-2 \pi k}}{1+e^{-2 \pi k}} .
$$

This shows that the Chandra-Singh transform of the univalent function $f$ is of infinite valence.

\section{References}

[1] J. W. Alexander II, Functions which map the interior of the unit circle upon simple regions, Ann. of Math. 17 (1915), 12-22.

[2] S. D. Bernardi, Convex and starlike univalent functions, Trans. Amer. Math. Soc. 135 (1969), 429-466.

[3] M. Biernacki, Sur l'intégrale des fonctions univalentes, Bull. Acad. Polon. Sci. Sér. Sci. Math. Astronom. Phys. 8 (1960), 29-34.

[4] D. M. Campbell and V. Singh, Valence properties of the solution of a differential equation, Pacific J. Math. 84 (1979), 29-33.

[5] S. Chandra and P. Singh, An integral transformation of some classes of regular univalent functions, Indian J. Pure Appl. Math. 6 (1975), 1270-1275.

[6] P. L. Duren, Univalent Functions, Springer, New York, 1983.

[7] D. J. Hallenbeck and T. H. MacGregor, Linear Problems and Convexity Techniques in Geometric Function Theory, Monogr. Stud. Math. 22, Pitman, Boston, 1984.

[8] J. Krzyż and Z. Lewandowski, On the integral of univalent functions, Bull. Acad. Polon. Sci. Sér. Sci. Math. Astronom. Phys. 11 (1963), 447-448.

[9] R. J. Libera, Some classes of regular univalent functions, Proc. Amer. Math. Soc. 16 (1965), 755-758.

[10] A. Marx, Untersuchungen über schlichte Abbildungen, Math. Ann. 107 (1933), 4067.

[11] Ch. Pommerenke, On close-to-convex analytic functions, Trans. Amer. Math. Soc. 114 (1965), 176-186.

[12] S. Ponnusamy and F. Rønning, Integral transforms of functions with the derivative in a halfplane, Israel J. Math. 114 (1999), 177-188.

[13] S. Ruscheweyh and T. Sheil-Small, Hadamard products of schlicht functions and the Pólya-Schoenberg conjecture, Comment. Math. Helv. 48 (1973), 119-135.

[14] R. Singh and S. Singh, Starlikeness and convexity of certain integrals, Ann. Univ. Mariae Curie-Skłodowska Sect. A 35 (1981), 145-148.

Department of Mathematics

Purdue University

West Lafayette, IN 47907, U.S.A.

E-mail: jeb@math.purdue.edu

Received 6.10.2006

and in final form 17.1.2007 